\title{
Predicting Tipping Points in Chaotic Maps with Period-Doubling Bifurcations
}

\author{
Changzhi Li, ${ }^{1}$ Dhanagopal Ramachandran, ${ }^{2}$ Karthikeyan Rajagopal $\mathbb{D},{ }^{3}$ Sajad Jafari, ${ }^{4,5,6}$ \\ and Yongjian Liu ${ }^{1}$ \\ ${ }^{1}$ Guangxi Colleges and Universities Key Laboratory of Complex System Optimization and Big Data Processing, \\ Yulin Normal University, Yulin 537000, China \\ ${ }^{2}$ Center for System Design, Chennai Institute of Technology, Chennai, India \\ ${ }^{3}$ Center for Nonlinear Systems, Chennai Institute of Technology, Chennai, India \\ ${ }^{4}$ Center for Computational Biology, Chennai Institute of Technology, Chennai, India \\ ${ }^{5}$ Biomedical Engineering Department, Amirkabir University of Technology, Tehran 15875-4413, Iran \\ ${ }^{6}$ Health Technology Research Institute, Amirkabir University of Technology, Hafez Ave, No. 350, Valiasr Square, \\ Tehran 159163-4311, Iran
}

Correspondence should be addressed to Karthikeyan Rajagopal; rkarthiekeyan@gmail.com

Received 18 March 2021; Accepted 24 May 2021; Published 4 June 2021

Academic Editor: Guillermo Huerta Cuellar

Copyright (C) 2021 Changzhi Li et al. This is an open access article distributed under the Creative Commons Attribution License, which permits unrestricted use, distribution, and reproduction in any medium, provided the original work is properly cited.

In this paper, bifurcation points of two chaotic maps are studied: symmetric sine map and Gaussian map. Investigating the properties of these maps shows that they have a variety of dynamical solutions by changing the bifurcation parameter. Sine map has symmetry with respect to the origin, which causes multistability in its dynamics. The systems' bifurcation diagrams show various dynamics and bifurcation points. Predicting bifurcation points of dynamical systems is vital. Any bifurcation can cause a huge wanted/unwanted change in the states of a system. Thus, their predictions are essential in order to be prepared for the changes. Here, the systems' bifurcations are studied using three indicators of critical slowing down: modified autocorrelation method, modified variance method, and Lyapunov exponent. The results present the efficiency of these indicators in predicting bifurcation points.

\section{Introduction}

Chaotic dynamics is interesting in the field of nonlinear systems. Real systems can present chaotic oscillations [1]. Phenomenological behaviors of chaotic systems are interesting [2]. Two types of systems can show chaos: continuous systems (flows) and discrete systems (maps) [3-5]. Chaos is still a challenge, and there are many unknown mysteries about it in both continuous and discrete chaotic systems [6-8]. Studying various dynamics of discrete and continuous systems has been a hot topic [9-11]. Hyperchaotic dynamics of coupled systems was discussed in [12]. A chaotic system with symmetry was investigated in [13]. A piecewise linear system was studied in $[14,15]$. Discrete systems can show many exciting dynamics, while most dynamics can be investigated using an in-depth study of their structures [16].
The reliability of the dynamics of discrete systems is highly dependent on simulation time [17]. The finite precision of computers has a significant effect on the simulation of chaotic dynamics [18]. Critical points of the bifurcation diagram in a chaotic map were investigated in [19]. Chaotic maps have some engineering applications, such as random number generators [20, 21]. In [22], the Henon map was investigated using fuzzy logic. Fractional order of the generalized Henon map was discussed in [23]. Various dynamics of the Bogdanov map were investigated in [24]. Multistability is an interesting behavior of dynamical systems $[15,25,26]$. Multistability is a condition in which the system's attractor is dependent on the initial values [27]. Various types of multistability can be discussed, such as extreme multistability [28]. Multistability of a 1D chaotic map has been studied in [29]. Secure communication and 
image encryption are some of the applications of chaotic dynamics [30, 31]. Image encryption based on the Bogdanov map is very applicable $[32,33]$. Control is an important challenge in the study of chaotic dynamics [34-36].

Dynamical properties of systems can be investigated using bifurcation diagrams. In a bifurcation diagram, various dynamics of the system can be seen as well as its bifurcation points [37-39]. Nonlinear dynamical tools are very useful $[40,41]$. Hidden and nonstandard bifurcations of a system were studied in [42]. The study of bifurcations of a nonautonomous memristive FitzHugh-Nagumo circuit has been done in [43]. In [44], bifurcations of memristor synapse-based Morris-Lecar were discussed. Recently, the study of bifurcation points and their predictions is interesting $[45,46]$. Before the occurrence of bifurcation points, slowing down is seen in the system dynamics [47]. This slowness is useful in the indication of bifurcation points [48, 49]. Prediction of bifurcation points is vital since some bifurcations may cause an undesired new behavior. Prediction of bifurcation points of biological systems has been studied in [50]. For example, the application of slowing down of blood pressure in predicting ischemic stroke was discussed in [51]. The ability of older adults to recover was investigated as a key for antiaging issue [52]. The advantage of predicting bifurcation points using indicators is to predict approaching the bifurcation points before their occurrence. Many studies try to indicate bifurcation points [53,54]. Prediction of noise-induced critical transitions was studied in [55]. The most exciting predictors of bifurcation points are autocorrelation at lag-1 and variance [47]. In [56], some issues in those indicators in predicting bifurcation points during a period-doubling route to chaos were studied. So, a new version of the well-known indicators was proposed to solve those issues [56]. In [57, 58], the Lyapunov exponent was studied as an indicator of bifurcation points. However, some points in the calculation of Lyapunov exponents should be considered [59].

Here, we study various dynamics of two chaotic maps. These maps show different bifurcations. The cobweb plot is used to study the dynamics of the chaotic map in which the transition of the time series is also shown in the map plot [60]. Then, using critical slowing down indicators, various tipping points of the systems are investigated.

\section{The Chaotic Maps}

Two chaotic maps are studied in this paper. The first one is a one-dimensional chaotic map, which is called the sine map, as shown in the following equation:

$$
x_{k+1}=B \sin \left(\pi x_{k}\right) .
$$

To study various behavior of the system by varying initial values, cobweb diagrams of the sine map in parameter $B=$ 0.6 and various initial conditions are shown in Figure 1. Here, in the cobweb plots, the map is shown in red color, the identity line is shown in cyan color, and the transition of time series is shown in black color. Figure 1 shows that the system has three equilibrium points in this parameter since its map has three intersections with the identity line where $x_{k+1}=x_{k}$. The origin is unstable because the slope of the map is larger than one, and the other ones are stable because the amplitude of slope is smaller than one. So, the system is multistable in this parameter, and initial conditions are crucial in the system's final state. The figure shows that different initial conditions result in various equilibrium points.

To briefly explain the dynamic of System (1), its bifurcation is presented in Figure 2. The diagram depicts that the system has various dynamics, and the amplitude of attractors is expanded by increasing the bifurcation parameter. The bifurcation diagram shows that the map has various dynamics from equilibrium points to chaotic attractors. In bifurcation parameters $0<B<0.318$, the system shows one fixed point at zero. Figure 3(a) shows the cobweb plot with $B=0.2$ and initial condition $x_{0}=0.2$. By increasing parameter $B$, two equilibrium points are created, and the origin becomes unstable (Figure 3(b) with $B=0.5$ and $x_{0}=0.2$ ). So, the two equilibrium points are coexisting. After that, an increase in parameter $B$ causes a period-doubling route to chaos. The cobweb plot of the chaotic dynamics with $B=$ 1 and $x_{0}=0.2$ is shown in Figure 3(c). So, in this interval of parameter $B$, the system has two stabilities, which can vary from an equilibrium point to chaos by changing the bifurcation parameter. By increasing parameter $B$, a crisis happens, and the chaotic attractor is expanded. Figure 3(d) shows that the attractor can move from the positive part of the sine function to the negative one and vice versa ( $B=1.001$ and $x_{0}=0.2$ ). After that, in $B=1.466$, a periodtwo dynamic is generated, followed by the period-doubling route to chaos. Figure 3(e) shows the cobweb plot of the system in $B=1.5$ and $x_{0}=0.2$ in which the system has a periodic dynamic. In larger parameters such as $B=2.5$ (Figure 3(f)), an exciting dynamic appears in which increasing parameter $B$ causes another peak of the sine function touches the identity line. It can create four new equilibrium points. Two equilibrium points are stable, and another two equilibrium points are unstable. Then, the same route to chaos happens by increasing parameter $B$, and the bifurcations repetitively happen, which are just different in the amplitude of the dynamics.

The sine function is symmetric concerning the origin. This property causes two different symmetric stabilities shown in the bifurcation diagram with two positive and negative initial conditions. Figure 4 shows the bifurcation diagram of System (1) with $x_{0}=0.5$ for the black diagram and $x_{0}=-0.5$ for the red diagram. The system has two stable equilibria in $B \in[0.317,0.719]$, and each of them continues with a period-doubling route to chaos. Also, in each of the periodic windows, the multistability of the system can be seen.

The second studied system is the Gaussian map. This map is formulated as follows:

$$
x_{k+1}=e^{-a x_{k}^{2}}+B,
$$

where parameter $a=6.2$ is fixed and parameter $B$ is the bifurcation parameter. Figure 5 shows bifurcation diagram 


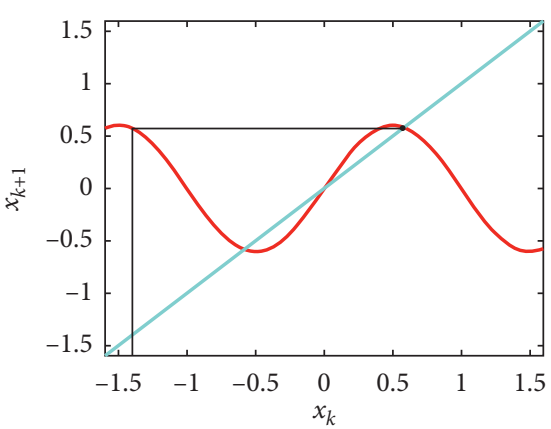

(a)

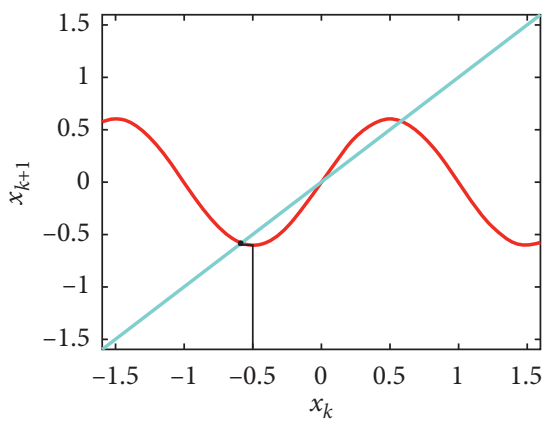

(d)

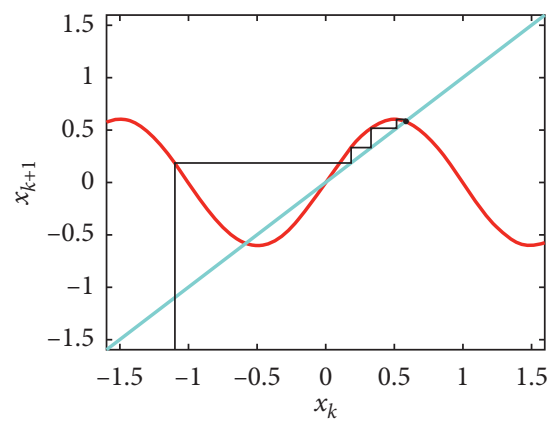

(b)

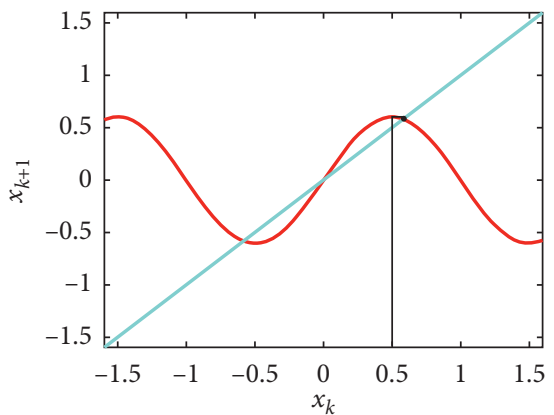

(e)

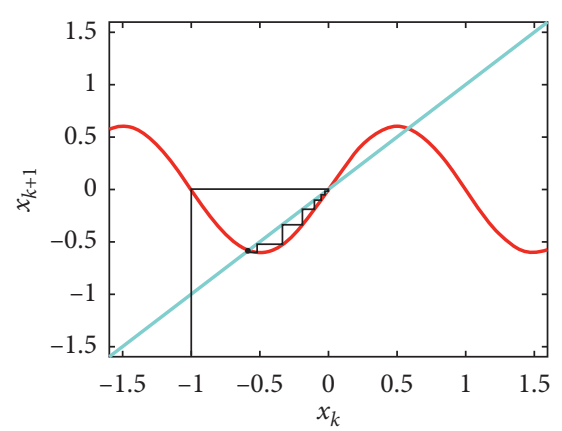

(c)

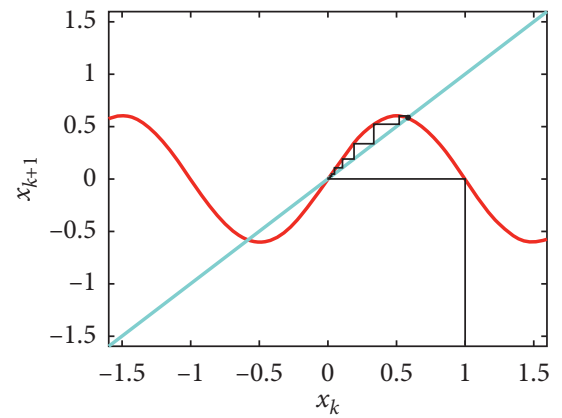

(f)

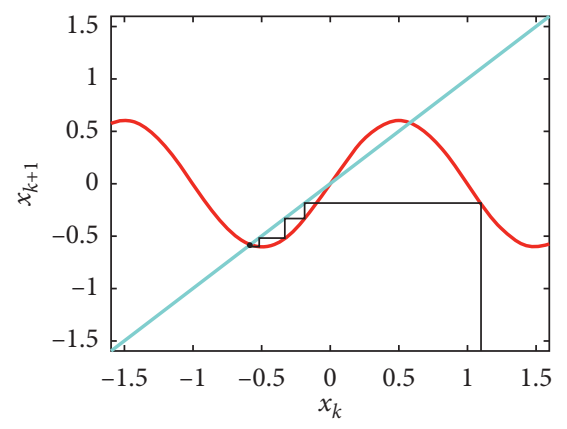

(g)

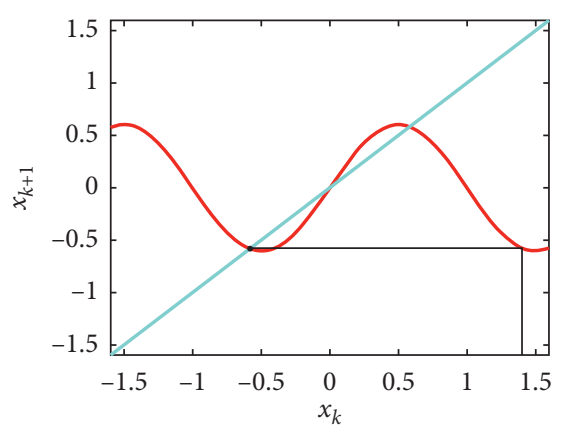

(h)

Figure 1: Cobweb diagram of System (1) in parameter $B=0.6$ and initial condition (a) $x_{0}=-1.4$; (b) $x_{0}=-1.1$; (c) $x_{0}=-1$; (d) $x_{0}=-0.5$; (e) $x_{0}=0.5$; (f) $x_{0}=1$; (g) $x_{0}=1.1$; (h) $x_{0}=1.4$; this shows that the system has two stable equilibria in this value of parameter.

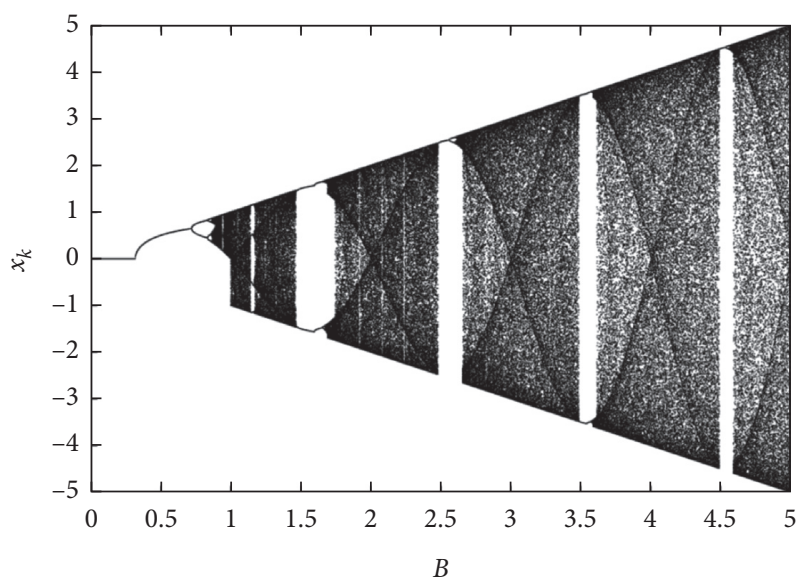

FIGURE 2: Bifurcation diagram by varying parameter $B$ and constant initial condition $x_{0}=0.5$. The symmetric sine map has a perioddoubling route to chaos and with periodic windows observed within. 


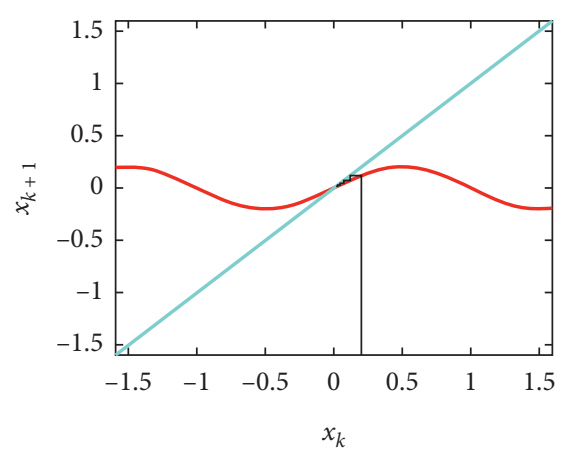

(a)

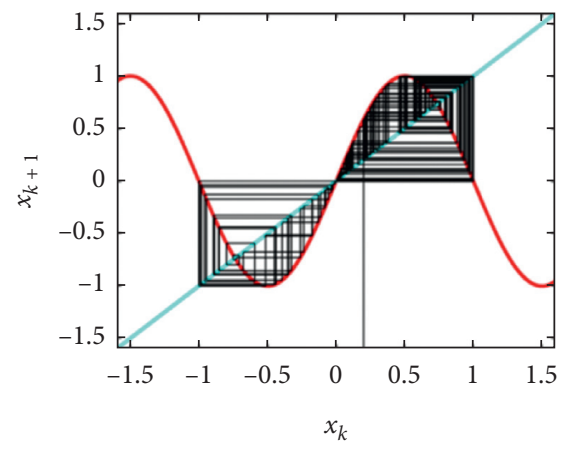

(d)

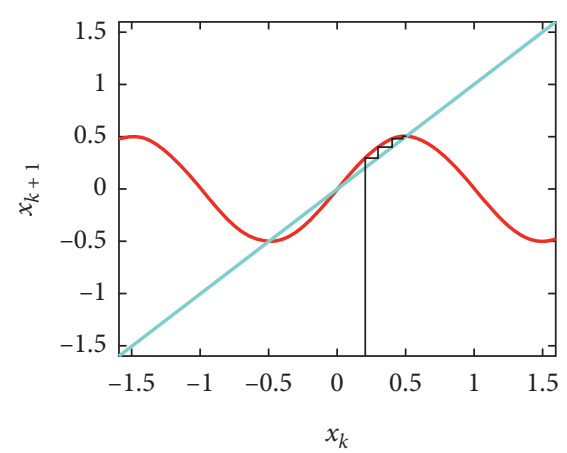

(b)

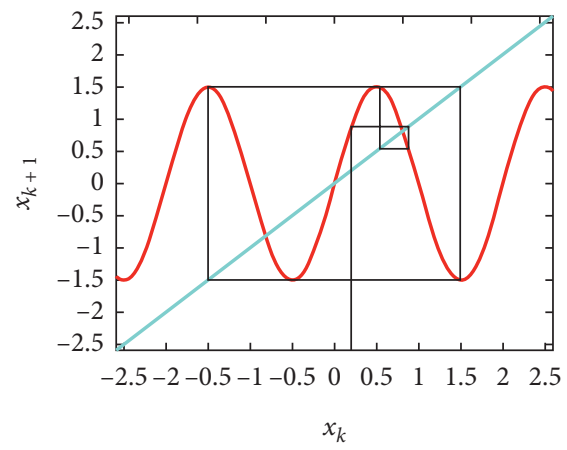

(e)

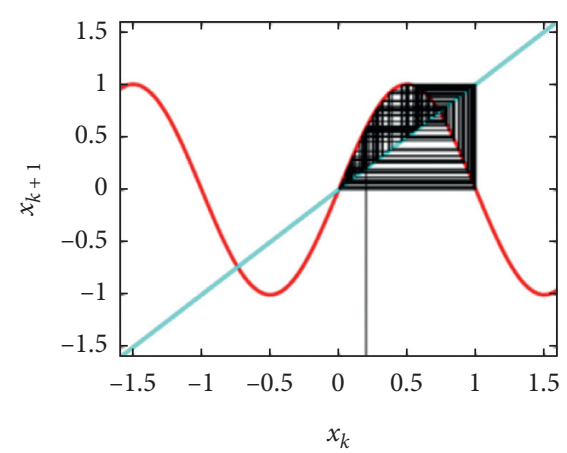

(c)

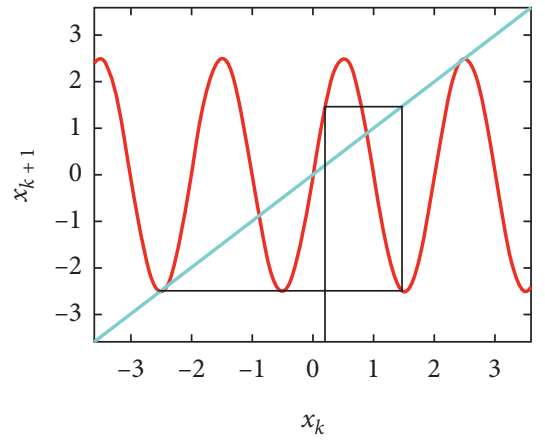

(f)

Figure 3: Cobweb diagram of System (1) in initial condition $x_{0}=0.2$ and parameter (a) $B=0.2 ;$ (b) $B=0.5 ;$ (c) $B=1$; (d) $B=1.001$; (e) $B=1.5$; (f) $B=2.5$; by increasing parameter $B$, the sine map changes from equilibrium points to chaos, and then increasing the parameter causes some periodic dynamics in periodic windows.

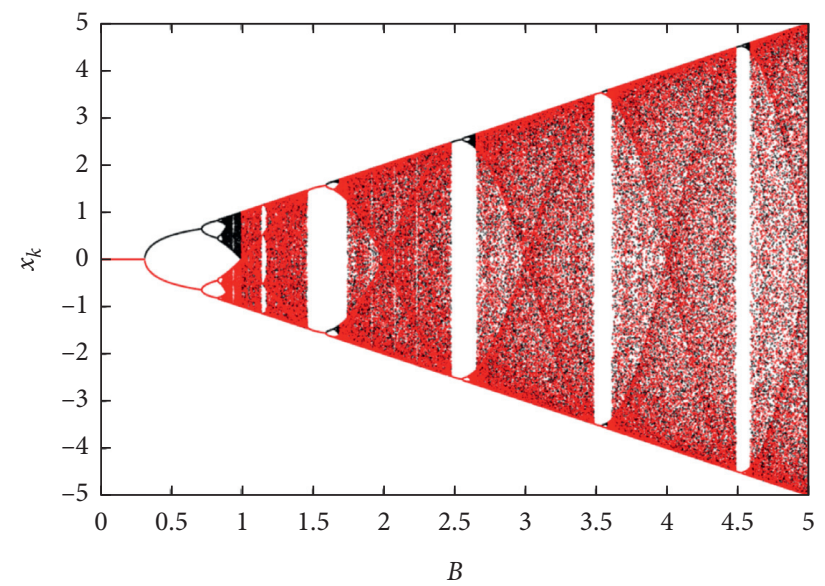

Figure 4: Bifurcation diagram by changing parameter $B$; the black diagram is plotted with $x_{0}=0.5$, and the red diagram is plotted with $x_{0}=-0.5$; the sine map has two stabilities in some intervals of parameter $B$, while in some other intervals, the two stabilities merge to one attractor.

of this map by changing parameter $B$. It can be seen that the system shows a period-doubling route to chaos and its inverse route by increasing $B$.

\section{Critical Slowing Down Indicators of the Chaotic Maps}

Here, the bifurcations of the symmetric sine map are studied. The studies of the previous section show that the system has various dynamics and many bifurcation points. Critical slowing down is observed before the tipping points. The slowness can be characterized using the critical slowing down predictors [47]. One of the useful slowing down indicators is autocorrelation at lag-1. Before the tipping points, the system became slower, so the similarity of consecutive states increases. Another well-known indicator is variance, which increases before the bifurcation points. However, a previous study has shown that these indicators can only 


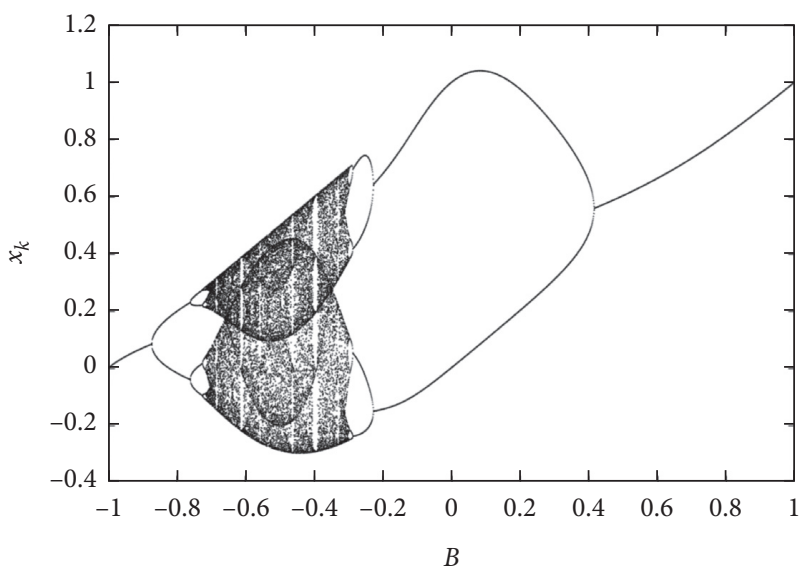

Figure 5: Bifurcation diagram of the Gaussian map by varying parameter $B$ and constant initial condition $x_{0}=0$. The system shows various dynamics in its period-doubling route.

predict tipping points of type "period-one" [56]. In that work, to modify the previous indicators, the previous indicators such as autocorrelation and variance were applied to subvectors obtained from each cycle of the $m$-cycle attractor where $m$ is the period of the signal [56].

The modified autocorrelation (AC) method is applied to the dynamics of the map for varying parameter $B$. Figure 6 shows the bifurcation diagram of the sine map in the interval $B \in[0,5]$ in black and the absolute of the modified autocorrelation indicator in blue. The results depict that the predictor indicates various bifurcation points in the route of period-doubling to chaos. Many bifurcations can be seen in this interval. For example, in $B=1.596$, a pitchfork bifurcation happens, which is predicted by increasing AC's value. Also, bifurcation points of the period-doubling route to chaos in the periodic window intervals such as $B \in[2.48,2.68]$ were appropriately predicted. To have a closer look at the performance of this predictor, Figure 7 (a) presents the bifurcation diagram and its correspondence modified autocorrelation in interval $B \in[0,1.2]$. By approaching the tipping point, the absolute of modified autocorrelation approaches its maximum value (one). Then, by going far away from the bifurcation point, its value decreases to zero. Figure 7(b) shows the estimated period of the system using the algorithm proposed in [56].

The modified variance method is applied to the dynamics of System (1) (Figure 8). In Figure 8(a), the bifurcation diagram is shown in black color, and the scaled version of the logarithm of the modified variance $(\times 0.1)$ is shown in blue color by changing $B \in[0,5]$. To have a closer view, these diagrams are shown in $B \in[0,1.2]$, in Figure $8(\mathrm{~b})$. The figure shows that the modified variance increases by approaching the bifurcation point and decreases by going far away from it.

Another indicator of bifurcation points is the Lyapunov exponent [57, 58]. Lyapunov exponent goes to zero by approaching the bifurcation points. It has an exact value in various bifurcation points. Figure 9 presents the bifurcation diagram in black and Lyapunov exponent in blue color.

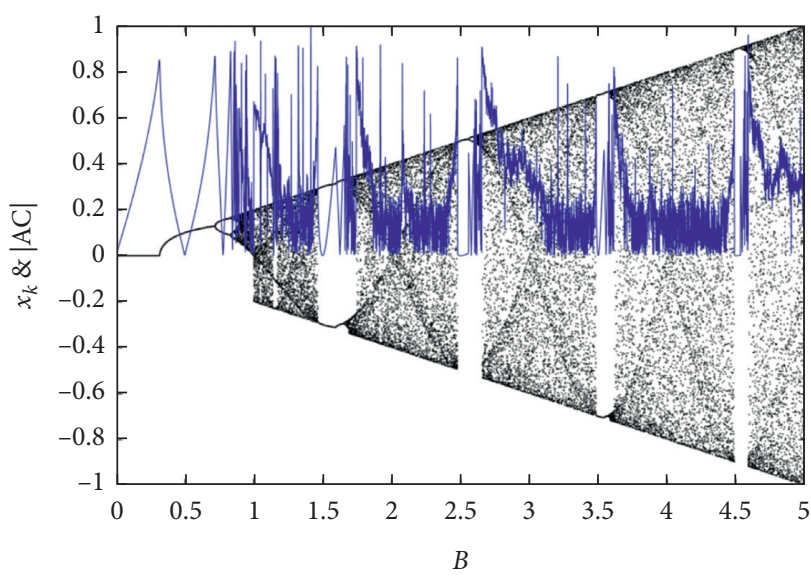

FIgURE 6: The scaled bifurcation diagram of the sine map $(\times 0.2)$ for changing $B \in[0,5]$ and initial condition $x_{0}=0.5$ in black and the absolute value of modified autocorrelation in blue color; this presents that $|A C|$ can predict various bifurcations by approaching to the value 1 .

Figure 9(a) shows the diagrams in $B \in[0,5]$ and Figure 9(b) shows in $B \in[0,1.2]$. The results depict that the Lyapunov exponent can predict various tipping points of the sine map.

The second studied system is the Gaussian map. It was shown that the system has various dynamics and bifurcation points. To predict the bifurcations, AC, variance, and Lyapunov exponent are used. Figure 10 shows that the absolute value of $\mathrm{AC}$ predicts various bifurcation points. For instance, the system has a bifurcation point in $B=-0.88$ from period- 1 to period- 2 dynamics. The absolute value of $\mathrm{AC}$ increases until its value becomes one in the bifurcation point. The same trends can be seen in other bifurcation points.

Figure 11 shows the modified variance, which is calculated from the states of the Gaussian map by changing parameter $B$. The figure shows that the variance increases before bifurcation points, but it does not reach a constant value in various bifurcation points. So, increasing variance alarms the approaching bifurcation points. 


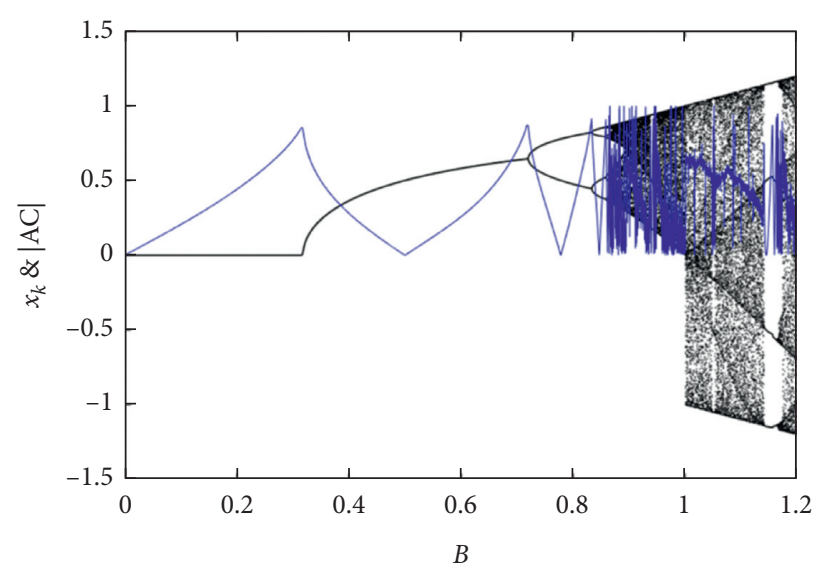

(a)

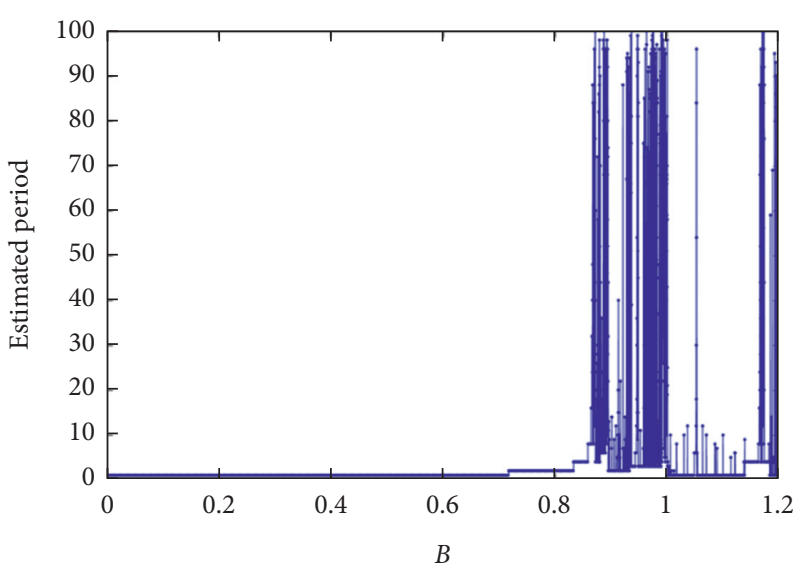

(b)

Figure 7: (a) The bifurcation diagram of the sine map by changing $B \in[0,1.2]$ and initial condition $x_{0}=0.5$ in black and the absolute value of modified autocorrelation in blue color; (b) the estimated period of the sine map in $B \in[0,1.2]$. The zoomed view shows better the ability of $|A C|$ to predict various bifurcations.

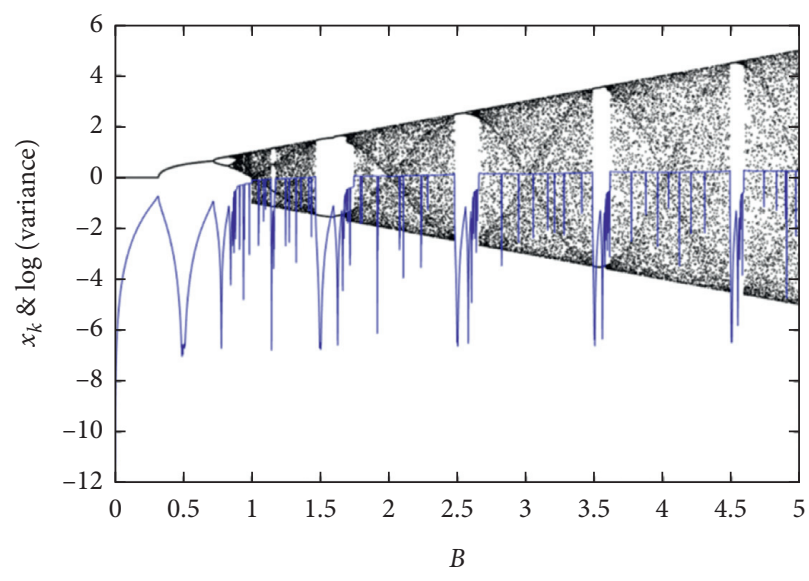

(a)

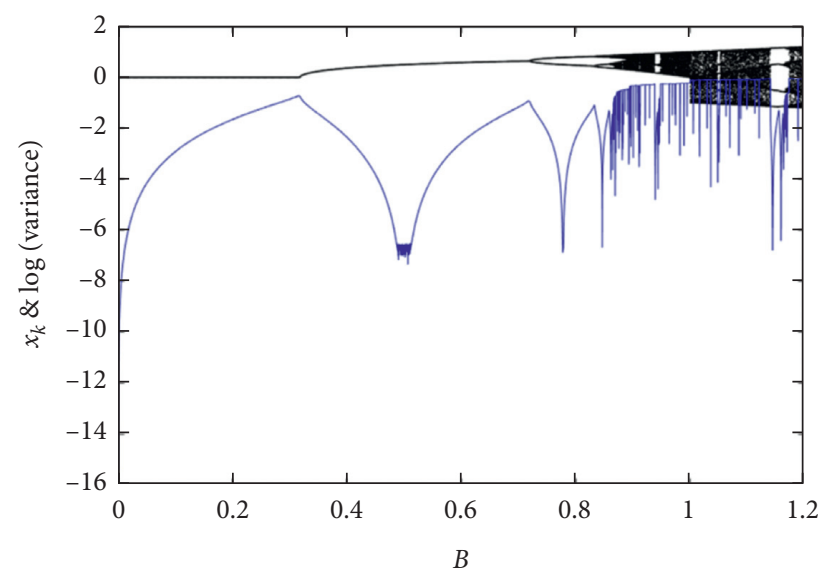

(b)

FIGURE 8: Bifurcation diagram in black color and the scaled version of the logarithm of the modified variance $(\times 0.1)$ in blue color (a) by changing $B \in[0,5]$; (b) by changing $B \in[0,1.2]$; the results show that the variance method increases by approaching bifurcations which alarms that a tipping point is getting close.

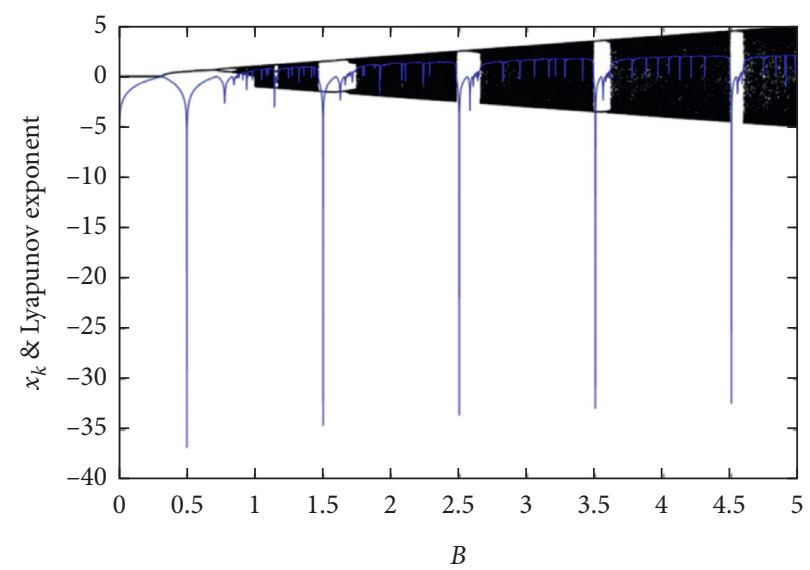

(a)

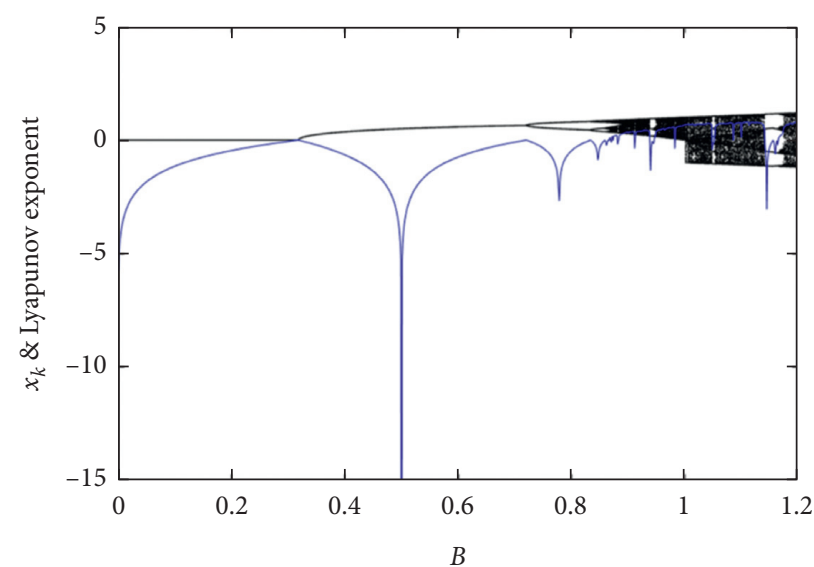

(b)

Figure 9: Bifurcation diagram in black and Lyapunov exponent in blue color by changing (a) $B \in[0,5]$; (b) $B \in[0,1.2]$; Lyapunov exponent approaches to zero by approaching bifurcation points, which can help their predictions. 


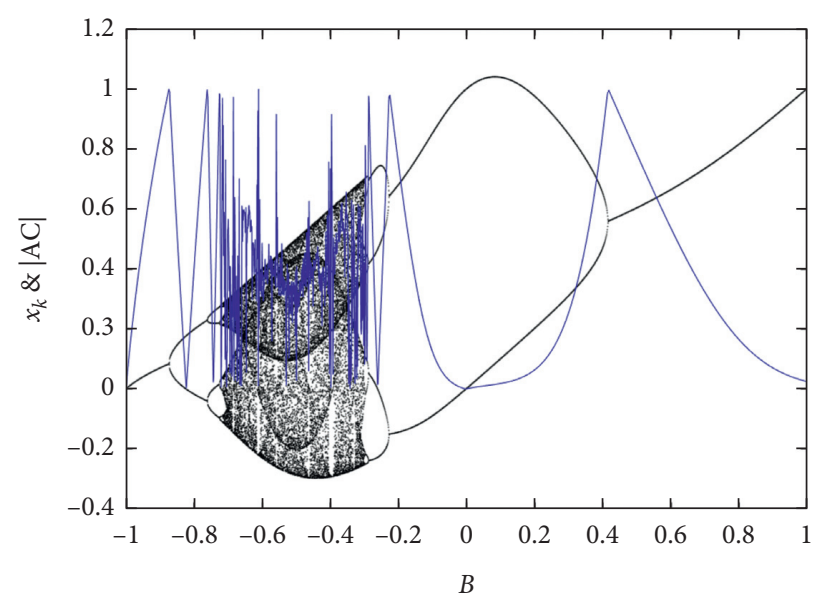

FIgURE 10: The bifurcation diagram of the Gaussian map with respect to changing $B \in[-1,1]$ and initial condition $x_{0}=0$ in black and the absolute value of modified autocorrelation in blue color; this presents that $|A C|$ can predict various bifurcations by approaching the value 1 .

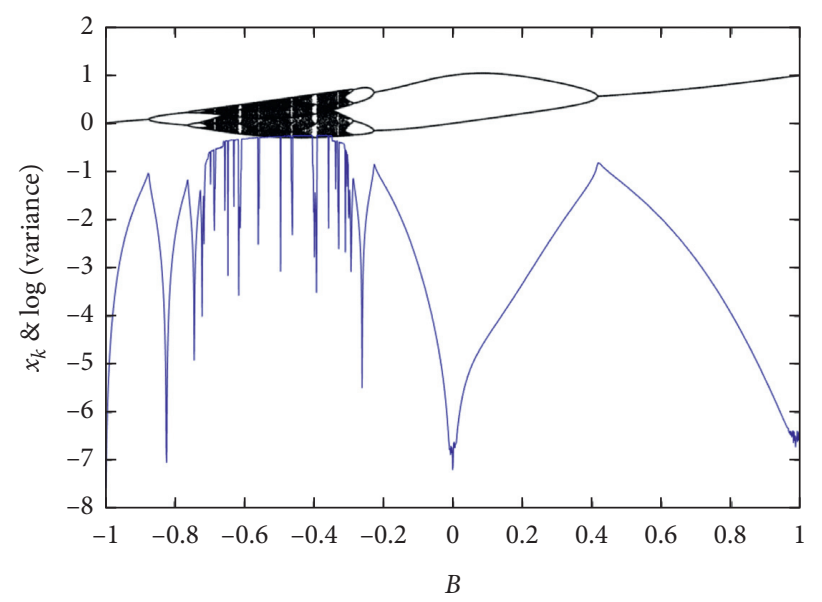

FiguRE 11: Bifurcation diagram in black color and the scaled version of the logarithm of the modified variance $(\times 0.1)$ in blue color by changing $B \in[-1,1]$; the results show that the variance method increases by approaching bifurcations, which alarms that tipping points are getting close.

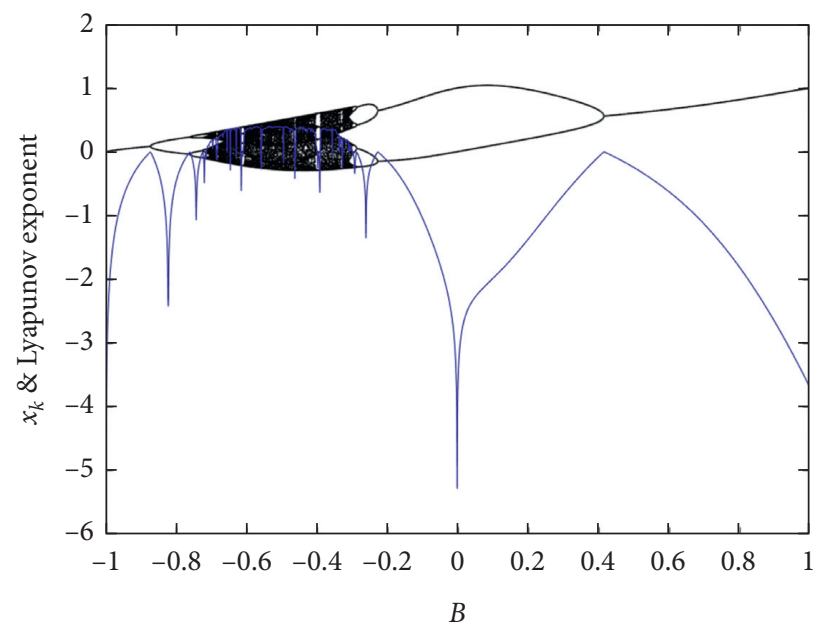

FiguRE 12: Bifurcation diagram in black and Lyapunov exponent in blue color by changing $B \in[-1,1]$; Lyapunov exponent approaches zero by approaching bifurcation points, which can help their predictions. 
Another studied indicator of the paper is the Lyapunov exponent. The results of the Lyapunov exponent are shown in Figure 12. Lyapunov exponent alarms approaching the bifurcation points by approaching zero. So, approaching zero shows that a bifurcation point is very close, while going away from it shows that the system is getting far from bifurcation points.

\section{Discussion and Conclusion}

This paper aimed to predict bifurcation points of chaotic maps. Two systems were studied: sine map and Gaussian map. Various dynamics of the sine map were studied, and its bifurcations were investigated. The results presented the multistability of the system because of its symmetric map. The system's critical slowing down was indicated using the modified autocorrelation method, modified variance method, and Lyapunov exponent. These studies showed that the bifurcation points of the sine map could be predicted using the indicators. The other studied system was the Gaussian map. The system shows various dynamics in a period-doubling route to chaos. Bifurcation points of the system were predicted using modified autocorrelation, modified variance, and Lyapunov exponent. Some indicators, such as autocorrelation and Lyapunov exponent, have an exact value in the bifurcation points. So, approaching the exact values indicates approaching the bifurcations. However, the variance method does not have an exact value in the bifurcation points. In the variance method, increasing the variance shows approaching the bifurcation points; however, we cannot precisely determine when it happens. The chaotic attractors are very complex dynamics. In chaotic domain, we cannot determine the transient time and slowness of dynamics. So, we cannot trust the indicators in the chaotic dynamics.

This study shows that some indications can alarm approaching the various bifurcation points. Those indicators had various natures. For example, autocorrelation calculates the short-term memory of the time series. Variance is based on the variations which increase by approaching the bifurcation points. The third method was the Lyapunov exponent, which shows the speed of the system approaching its final dynamic. The results, tested on two discrete systems, showed that these indicators had a proper trend when approaching bifurcation points that alarms their occurrences. In the future works, prediction of bifurcation points of systems with other types of bifurcations than perioddoubling route to chaos can be investigated. As a suggestion for future works, some can consider the application of deep learning and reinforcement learning in the prediction of tipping points.

\section{Data Availability}

All the numerical simulation parameters are mentioned in the respective text part, and there are no additional data requirements for the simulation results.

\section{Conflicts of Interest}

The authors declare that they have no conflicts of interest to report regarding the present study.

\section{Acknowledgments}

This work was supported by the High Level Innovation Team Program from Guangxi Higher Education Institutions of China (Document No. [2018] 35). This work was funded by the Center for Computational Biology, Chennai Institute of Technology, India (funding no. CIT/CCB/2021/RD/007).

\section{References}

[1] G. Huerta-Cuellar, E. Jiménez-López, E. Campos-Cantón, and A. N. Pisarchik, "An approach to generate deterministic Brownian motion," Communications in Nonlinear Science and Numerical Simulation, vol. 19, no. 8, pp. 2740-2746, 2014.

[2] A. N. Pisarchik, R. Jaimes-Reátegui, R. Sevilla-Escoboza, G. Huerta-Cuellar, and M. Taki, "Rogue waves in a multistable system," Physical Review Letters, vol. 107, no. 27, p. 274101, 2011.

[3] E. Mliki, N. Hasanzadeh, F. Nazarimehr, A. Akgul, O. Boubaker, and S. Jafari, "Some new chaotic maps with application in stochastic," in Recent Advances in Chaotic Systems and Synchronization, pp. 165-185, Elsevier, Amsterdam, Netherlands, 2019.

[4] R. Lozi and A. N. Pchelintsev, "A new reliable numerical method for computing chaotic solutions of dynamical systems: the Chen attractor case," International Journal of Bifurcation and Chaos, vol. 25, no. 13, p. 1550187, 2015.

[5] N. Wang, G. Zhang, and H. Bao, "A simple autonomous chaotic circuit with dead-zone nonlinearity," IEEE Transactions on Circuits and Systems II: Express Briefs, vol. 67, no. 12, pp. 3502-3506, 2020.

[6] F. Nazarimehr, S. Jafari, G. Chen et al., "A tribute to J. C. Sprott," International Journal of Bifurcation and Chaos, vol. 27, no. 14, p. 1750221, 2017.

[7] J. L. Echenausía-Monroy and G. Huerta-Cuéllar, "A novel approach to generate attractors with a high number of scrolls," Nonlinear Analysis: Hybrid Systems, vol. 35, p. 100822, 2020.

[8] N. Wang, G. Zhang, N. V. Kuznetsov, and H. Bao, "Hidden attractors and multistability in a modified Chua's circuit," Communications in Nonlinear Science and Numerical Simulation, vol. 92, p. 105494, 2021.

[9] R. Lozi, V. A. Pogonin, and A. N. Pchelintsev, "A new accurate numerical method of approximation of chaotic solutions of dynamical model equations with quadratic nonlinearities," Chaos, Solitons \& Fractals, vol. 91, pp. 108-114, 2016.

[10] J. Echenausía-Monroy, J. H. García-López, R. JaimesReátegui, and G. Huerta-Cuellar, "Evidence of multistability in a multiscroll generator system," Advances on Nonlinear Dynamics of Electronic Systems, vol. 17, 2019.

[11] G. D. Leutcho, J. Kengne, L. Kamdjeu Kengne, A. Akgul, V.-T. Pham, and S. Jafari, "A novel chaotic hyperjerk circuit with bubbles of bifurcation: mixed-mode bursting oscillations, multistability, and circuit realization," Physica Scripta, vol. 95, no. 7, Article ID 075216, 2020.

[12] R. J. Escalante-González and E. Campos, "Hyperchaotic attractors through coupling of systems without equilibria," 
The European Physical Journal Special Topics, vol. 229, no. 6-7, pp. 1309-1318, 2020.

[13] G. D. Leutcho and J. Kengne, "A unique chaotic snap system with a smoothly adjustable symmetry and nonlinearity: chaos, offset-boosting, antimonotonicity, and coexisting multiple attractors," Chaos, Solitons \& Fractals, vol. 113, pp. 275-293, 2018.

[14] E. Campos, "Derivation of a continuous time dynamic planar system with two unstable foci from a three-dimensional chaotic piecewise linear system," Chaos: An Interdisciplinary Journal of Nonlinear Science, vol. 30, no. 5, Article ID 053114, 2020.

[15] R. Escalante-González and E. Campos, "Multistable systems with hidden and self-excited scroll attractors generated via piecewise linear systems," Complexity, vol. 2020, 2020.

[16] F. Nazarimehr, S. Jafari, S. M. R. H. Golpayegani, and L. H. Kauffman, "Investigation of bifurcations in the process equation," International Journal of Bifurcation and Chaos, vol. 27, no. 13, p. 1750201, 2017.

[17] E. G. Nepomuceno, S. A. M. Martins, B. C. Silva, G. F. V. Amaral, and M. Perc, "Detecting unreliable computer simulations of recursive functions with interval extensions," Applied Mathematics and Computation, vol. 329, pp. 408-419, 2018.

[18] T. E. Nazaré, E. G. Nepomuceno, S. A. M. Martins, and D. N. Butusov, "A note on the reproducibility of chaos simulation," Entropy, vol. 22, no. 9, p. 953, 2020.

[19] M. Romera, G. Pastor, M.-F. Danca et al., "Bifurcation diagram of a map with multiple critical points," International Journal of Bifurcation and Chaos, vol. 28, no. 5, p. 1850065, 2018.

[20] A. Beirami, H. Nejati, and W. H. Ali, "Zigzag map: a variability-aware discrete-time chaotic-map truly random number generator," Electronics Letters, vol. 48, no. 24, pp. 1537-1538, 2012.

[21] F. J. Farsana and K. Gopakumar, "A novel approach for speech encryption: zaslavsky map as pseudo random number generator," Procedia Computer Science, vol. 93, pp. 816-823, 2016.

[22] D. Cafagna and G. Grassi, "Decomposition method for studying smooth Chua's equation with application to hyperchaotic multiscroll attractors," International Journal of Bifurcation and Chaos, vol. 17, no. 1, pp. 209-226, 2007.

[23] L. Jouini, A. Ouannas, A.-A. Khennaoui, X. Wang, G. Grassi, and V.-T. Pham, "The fractional form of a new three-dimensional generalized Hénon map," Advances in Difference Equations, vol. 2019, p. 122, 2019.

[24] D. K. Arrowsmith, J. H. E. Cartwright, A. N. Lansbury, and C. M. Place, "The Bogdanov map: bifurcations, mode locking, and chaos in a dissipative system," International Journal of Bifurcation and Chaos, vol. 3, no. 4, pp. 803-842, 1993.

[25] H. E. Gilardi-Velázquez, L. J. Ontañón-García, D. G. Hurtado-Rodriguez, and E. Campos-Cantón, "Multistability in piecewise linear systems versus eigenspectra variation and round function," International Journal of Bifurcation and Chaos, vol. 27, no. 9, p. 1730031, 2017.

[26] J. L. Echenausía-Monroy, G. Huerta-Cuellar, R. JaimesReátegui et al., "Multistability emergence through fractionalorder-derivatives in a PWL multi-scroll system," Electronics, vol. 9, no. 6, p. 880, 2020.

[27] A. Anzo-Hernández, H. E. Gilardi-Velázquez, and E. Campos-Cantón, "On multistability behavior of unstable dissipative systems," Chaos: An Interdisciplinary Journal of Nonlinear Science, vol. 28, no. 3, Article ID 033613, 2018.
[28] C. R. Hens, R. Banerjee, U. Feudel, and S. K. Dana, "How to obtain extreme multistability in coupled dynamical systems," Physical Review. E, Statistical, Nonlinear, and Soft Matter Physics, vol. 85, Article ID 035202, 2012.

[29] E. J. Staunton and P. T. Piiroinen, "Noise and multistability in the square root map," Physica D: Nonlinear Phenomena, vol. 380-381, pp. 31-44, 2018.

[30] A. Pisarchik, R. Jaimes-Reátegui, C. Rodríguez-Flores, J. García-López, G. Huerta-Cuellar, and F. Martín-Pasquín, "Secure chaotic communication based on extreme multistability," Journal of the Franklin Institute, vol. 358, no. 4, pp. 2561-2575, 2021.

[31] B. Cassal-Quiroga and E. Campos-Cantón, "Generation of dynamical S-boxes for block ciphers via extended logistic map," Mathematical Problems in Engineering, vol. 2020, 2020.

[32] S. V. Janardhanan and P. Sanjeeva, "Bogdanov map-based permuted double image encryption," Anais da Academia Brasileira de Ciencias, vol. 92, p. e20181207, 2020.

[33] R. N. R. Parida, S. Singh, and C. Pradhan, "Analysis of color image encryption using multidimensional Bogdanov map," in Histopathological Image Analysis in Medical Decision Making, pp. 202-225, IGI Global, Hershey, PA, USA, 2019.

[34] J. L. Echenausía-Monroy, J. H. García-López, R. JaimesReátegui, and G. Huerta-Cuéllar, "Parametric control for multiscroll generation: electronic implementation and equilibrium analysis," Nonlinear Analysis: Hybrid Systems, vol. 38, p. 100929, 2020.

[35] J. L. Echenausía-Monroy, J. H. García-López, R. JaimesReátegui, and G. Huerta-Cuellar, "Electronic implementation dataset to monoparametric control the number of scrolls generated," Data in Brief, vol. 31, p. 105992, 2020.

[36] N. Wang, G. Zhang, and H. Li, "Parametric control for multiscroll attractor generation via nested sine-PWL function," IEEE Transactions on Circuits and Systems II: Express Briefs, vol. 68, 2020.

[37] R. C. Hilborn, Chaos and Nonlinear Dynamics: An Introduction for Scientists and Engineers, Oxford University Press, Oxford, UK, 2000.

[38] H. Bao, Z. Hua, N. Wang, L. Zhu, M. Chen, and B. Bao, "Initials-boosted coexisting chaos in a 2-D sine map and its hardware implementation," IEEE Transactions on Industrial Informatics, vol. 17, no. 2, pp. 1132-1140, Feb 2021.

[39] Z. Wei, B. Zhu, J. Yang, M. Perc, and M. Slavinec, "Bifurcation analysis of two disc dynamos with viscous friction and multiple time delays," Applied Mathematics and Computation, vol. 347, pp. 265-281, 2019.

[40] S. M. Shekatkar, Y. Kotriwar, K. P. Harikrishnan, and G. Ambika, "Detecting abnormality in heart dynamics from multifractal analysis of ECG signals," Scientific Reports, vol. 7, pp. 15127-15211, 2017.

[41] M.-F. Danca and G. Chen, "Bifurcation and chaos in a complex model of dissipative medium," International Journal of Bifurcation and Chaos, vol. 14, no. 10, pp. 3409-3447, 2004.

[42] G. Pastor, M. Romera, M.-F. Danca et al., "Hidden and nonstandard bifurcation diagram of an alternate quadratic system," International Journal of Bifurcation and Chaos, vol. 26, no. 2, p. 1650036, 2016.

[43] M. Chen, J. Qi, H. Wu, Q. Xu, and B. Bao, "Bifurcation analyses and hardware experiments for bursting dynamics in non-autonomous memristive FitzHugh-Nagumo circuit," Science China Technological Sciences, vol. 63, pp. 1-10, 2020.

[44] H. Bao, D. Zhu, W. Liu, Q. Xu, M. Chen, and B. Bao, "Memristor synapse-based morris-lecar model: bifurcation analyses and FPGA-based validations for periodic and chaotic 
bursting/spiking firings," International Journal of Bifurcation and Chaos, vol. 30, no. 3, p. 2050045, 2020.

[45] F. Nazarimehr, S. M. R. Hashemi Golpayegani, and B. Hatef, "Does the onset of epileptic seizure start from a bifurcation point?” The European Physical Journal Special Topics, vol. 227, no. 7-9, pp. 697-705, 2018.

[46] M. Scheffer, J. Bascompte, W. A. Brock et al., "Early-warning signals for critical transitions," Nature, vol. 461, no. 7260 , pp. 53-59, 2009.

[47] M. Scheffer, S. R. Carpenter, T. M. Lenton et al., "Anticipating critical transitions," Science, vol. 338 , no. 6105 , pp. 344-348, 2012.

[48] I. A. Van De Leemput, M. Wichers, A. O. J. Cramer et al., "Critical slowing down as early warning for the onset and termination of depression," Proceedings of the National Academy of Sciences, vol. 111, no. 1, pp. 87-92, 2014.

[49] S. V. George, S. Kachhara, R. Misra, and G. Ambika, "Early warning signals indicate a critical transition in Betelgeuse," Astronomy \& Astrophysics, vol. 640, p. L21, 2020.

[50] M. Scheffer, J. E. Bolhuis, D. Borsboom et al., "Quantifying resilience of humans and other animals," Proceedings of the National Academy of Sciences, vol. 115, no. 47, pp. 1188311890, 2018.

[51] V.-V. Le, T. Mitiku, G. Sungar, J. Myers, and V. Froelicher, "The blood pressure response to dynamic exercise testing: a systematic review," Progress in Cardiovascular Diseases, vol. 51, no. 2, pp. 135-160, 2008.

[52] S. Ukraintseva, K. Arbeev, M. Duan et al., "Decline in biological resilience as key manifestation of aging: potential mechanisms and role in health and longevity," Mechanisms of Ageing and Development, vol. 194, p. 111418, 2021.

[53] V. Dakos, S. R. Carpenter, W. A. Brock et al., "Methods for detecting early warnings of critical transitions in time series illustrated using simulated ecological data," PLoS One, vol. 7, no. 7, Article ID e41010, 2012.

[54] P. M. Müller, J. Heitzig, J. Kurths, K. Lüdge, and M. Wiedermann, "Anticipation-induced social tipping--can the environment be stabilised by social dynamics?," 2020, https://arxiv.org/abs/2012.01977.

[55] J. Ma, Y. Xu, Y. Li, R. Tian, S. Ma, and J. Kurths, "Quantifying the parameter dependent basin of the unsafe regime of asymmetric Lévy-noise-induced critical transitions," Applied Mathematics and Mechanics, vol. 42, no. 1, pp. 65-84, 2021.

[56] F. Nazarimehr, S. Jafari, S. M. R. Hashemi Golpayegani, M. Perc, and J. C. Sprott, "Predicting tipping points of dynamical systems during a period-doubling route to chaos," Chaos: An Interdisciplinary Journal of Nonlinear Science, vol. 28, no. 7, Article ID 073102, 2018.

[57] F. Nazarimehr, S. Jafari, S. M. R. Hashemi Golpayegani, and J. C. Sprott, "Can Lyapunov exponent predict critical transitions in biological systems?" Nonlinear Dynamics, vol. 88, no. 2, pp. 1493-1500, 2017.

[58] F. Nazarimehr, A. Ghaffari, S. Jafari, and S. M. R. H. Golpayegani, "Sparse recovery and dictionary learning to identify the nonlinear dynamical systems: one step toward finding bifurcation points in real systems," International Journal of Bifurcation and Chaos, vol. 29, no. 3, p. 1950030, 2019.

[59] M. L. C. Peixoto, E. G. Nepomuceno, S. A. M. Martins, and M. J. Lacerda, "Computation of the largest positive Lyapunov exponent using rounding mode and recursive least square algorithm," Chaos, Solitons \& Fractals, vol. 112, pp. 36-43, 2018.
[60] R. Stoop and W.-H. Steeb, Berechenbares Chaos in Dynamischen Systemen, Springer-Verlag, Berlin, Germany, 2006. 